\title{
Complete clinical retention of sealant materials should not be contemplated as cut-off for clinical success
}

\section{Steffen MICKENAUTSCH Veeresamy YENGOPAL}

University of the Witwatersrand, Faculty of Health Sciences, Department of Community Dentistry, Johannesburg, South Africa.

Declaration of Interests: The authors certify that they have no commercial or associative interest that represents a conflict of interest in connection with the manuscript.

\section{Corresponding Author:}

Steffen Mickenautsch

E-mail: neem@global.co.za

DOI: 10.1590/1807-3107BOR-2016.vol30.0032

Submitted: Sep 29, 2015

Accepted for publication: Oct 14, 2015

Last revision: Nov 16, 2015

\section{Dear Editor,}

We read with interest the randomised clinical trial report by Hesse et al. ${ }^{1}$ concerning low-cost glass ionomer cement as ART sealant in permanent molars. In their Discussion, the authors state "... the choice of considering only fully retained sealants as successful is supported by the current theory that the complete clinical retention of sealant materials should be contemplated as the cutoff for clinical success."

To our surprise the authors support this statement by reference to our published systematic review on the validity of sealant retention as surrogate for caries prevention. ${ }^{2}$ Our surprise is based on the fact that our systematic review actually provides empirical evidence to the exact contrary: it clearly shows that the retention rate of fissure sealants cannot be regarded as a valid surrogate for caries prevention.

Valid surrogate endpoints that are clinically meaningful require compliance with the Prentice criterion: (i) the surrogate needs to correlate with its true clinical endpoint and (ii) the surrogate/clinical endpoint correlation needs to be independent from the treatment type applied. ${ }^{3}$ This means that for sealant retention to be a valid surrogate for caries prevention the material retention-loss risk should be directly associated with the caries risk and that such association holds true regardless whether the sealant was placed e.g. with composite resin or glass-ionomer. Our findings show a direct association for resin (but with only a low adjusted $R^{2}=0.28$ ) but not for glass-ionomer sealants and that the ratio of retention-loss risk to the risk of caries on sealed tooth surfaces was not sealant material independent. ${ }^{2}$

In addition, we established in another meta-epidemiological study, including 95 clinical trails, that the prediction of future caries on basis of retention-loss of resin-based sealants is no more accurate than mere random guesses (Statistical comparison of prediction accuracy of material retention loss versus random numbers: Wilcoxon test, $\mathrm{z}=0.56$; $\mathrm{p}=0.58 /$ Sign test, $\mathrm{z}=1.38 ; \mathrm{p}=0.58){ }^{4}$

For these reasons, complete retention of sealant materials, expressed as the retention rate, is thus (in terms of caries prevention as its true clinical endpoint) a meaningless outcome measure and its use should best be avoided, in order to prevent misleading conclusions as to the clinical efficacy of fissure sealant materials.

Complete retention of sealant materials should definitely not be contemplated as any cut-off for clinical success. 
- Complete clinical retention of sealant materials should not be contemplated as cut-off for clinical success

\section{References}

1. Hesse D, Bonifácio CC, Guglielmi CAB, Franca C, Mendes FM, Raggio DP. Low-cost glass ionomer cement as ART sealant in permanent molars: a randomized clinical trial. Braz Oral Res. 2015;29(1):1-9. doi:10.1590/1807-3107BOR-2015.vol29.0063

2. Mickenautsch S, Yengopal V. Validity of sealant retention as surrogate for caries prevention:a systematic review. PLoS One. 2013;8(10):e77103. doi:10.1371/journal.pone.0077103
3. Prentice RL. Surrogate endpoints in clinical trials: definition and operational criteria. Stat Med. 1989;8(4):431-40. doi:10.1002/sim.4780080407

4. Mickenautsch S, Yengopal V. Retention loss of resin based fissure sealants - a valid predictor for clinical outcome?. Open Dent J. 2013;7:102-8. doi:10.2174/18742106201305130001. eCollection 2013 